\title{
Apertura hacia la anticoncepción adolescente entre los padres y las madres en México: un estudio de caso desde la sociodemografía
}

\section{Openness of fathers and mothers toward adolescent contraception in Mexico: a case study from a socio-demographic perspective}

\author{
Norma Ojeda de la Peña, Raúl S. González-Ramírez \\ y Alina Ramos-Vargas \\ San Diego State University, Estados Unidos \\ El Colegio de la Frontera Norte, México \\ Universidad Autónoma de Baja California, México
}

\begin{abstract}
Resumen
Se analizan las actitudes de los padres y madres respecto de la sexualidad y la anticoncepción adolescente en México, mediante el caso de Tijuana, B.C. Se examinan los niveles de apertura de los padres de ambos sexos en favor de proporcionar información al respecto a los adolescentes de ambos sexos. Este es un estudio socio-demográfico y de tipo descriptivo basado en información de una encuesta a 2,547 padres y madres con hijos en escuelas secundarias públicas. Los resultados indican interesantes diferencias de género significativas, donde las madres presentan índices de apertura mayores que los padres. Otras variables significativas son la escolaridad y el tener antecedentes de maternidad adolescente en la historia reproductiva de las madres y de las esposas o compañeras de los padres varones.
\end{abstract}

Palabras clave: Sexualidad, anticoncepción, adolescentes, familia, género, México.

Abstract

This is an analysis of attitudes of fathers and mothers on adolescent sexuality and contraception use in Mexico through the case of Tijuana, B.C. The analysis focuses on the parental openness toward providing information on sexuality and contraception to adolescents of both sexes. This is a socio-demographic study. A descriptive statistical analysis is conducted using data gathered through a survey of 2,547 fathers and mothers with children in public junior high schools. Research findings indicate significant gender differences. Mothers have a higher openness index than fathers. Other statistically significant variables are parent's education and adolescent maternity background in the reproductive history of mothers and wives of fathers.

Key words: Sexuality, contraception, adolescents, family, gender, Mexico. 


\section{INTRODUCCIÓN}

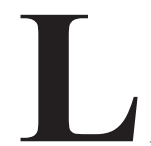

a práctica anticonceptiva de los adolescentes en México sigue siendo muy baja, según documenta la investigación demográfica realizada sobre el tema de manera reciente. Asimismo, la práctica sexual desprotegida entre la población mexicana, pero particularmente entre los y las más jóvenes, sigue siendo parte de la realidad que conlleva, entre otras cosas, a la ocurrencia de enfermedades de trasmisión sexual y embarazos a edades prematuras y frecuentemente no deseados. Fenómeno este último que casi siempre culmina en maternidad adolescente $\mathrm{y}$, frecuentemente, en paternidad adolescente. La aceptación social y, en contraparte, el costo social de este fenómeno sin duda ha cambiado al paso de las generaciones. Esto es conforme también han cambiado las condiciones macro sociales, económicas, demográficas y culturales en las que se dan. Hoy en día, podemos decir que, en lo general, tanto el embarazo como la maternidad adolescentes son fenómenos no del todo deseables entre amplios sectores de la población mexicana; sino que más bien se les prefiere evitar en lo posible. Sin embargo, siguen ocurriendo principalmente entre los grupos de la población socioeconómica más vulnerables, entre otras razones, como consecuencia directa de la falta de educación sexual y del limitado uso de anticonceptivos entre los adolescentes sexualmente activos. Situación que si bien está vinculada a múltiples factores, uno de particular importancia se refiere al limitado acceso que tienen los y las adolescentes a la información de calidad acerca de la sexualidad y la anticoncepción en los distintos ámbitos sociales en los éstos se desenvuelven. En este sentido, un ámbito de particular importancia es el familiar y el sustrato cultural correspondiente en el que se lleva a cabo la socialización de los niños y los adolescentes de ambos sexos en materia de género y sexualidad.

Conscientes de la importancia de este aspecto en la formación de los valores sociales y las ideas que adquieren los niños y los adolescentes en estos dos temas, el presente trabajo tiene por objetivo contribuir al análisis de la trasmisión intergeneracional de información sobre la sexualidad y la anticoncepción; específicamente de padres a hijos en el ámbito familiar. Para este propósito, se analiza el caso de un grupo de familias jóvenes de los sectores populares de la ciudad de Tijuana en Baja California, México. Concretamente, se analizan las actitudes o posturas de los padres de ambos sexos, con hijos en edad escolar secundaria, en favor de proporcionar 
Apertura hacia la anticoncepción adolescente entre los padres y las madres en México: un estudio de caso ... / N. OJEDA et al.

información acerca de la sexualidad y la anticoncepción a los adolescentes. Se parte del supuesto teórico que establece la sociología de los roles sociales acerca del proceso de socialización intergenracional en materia de género en el ámbito familiar.

Se trata de un estudio socio demográfico de tipo descriptivo basado en el análisis de frecuencias y estadística descriptivas. Asimismo, se calcula un "índice de apertura" que pretende medir el grado de apertura de los padres de ambos sexos en favor de la anticoncepción adolescente; así como su relación con algunas características sociales y demográficas de los padres y las madres. Esto último se realiza mediante un análisis de regresión logística de la razón de momios (odds ratio) de la probabilidad de tener una actitud más o menos abierta hacia la anticoncepción adolescente. Para este propósito se utiliza información proveniente de la "Encuesta a Padres de Familia con Hijos en Escuelas Secundarias, Tijuana, B.C., 2015”.

Se reconoce que el tema de este estudio es mucho más complejo de lo que puede ser captado y medido utilizando únicamente una metodología cuantitativa como la que aquí se propone. Idealmente este tema debería estudiarse utilizando una metodología mixta que permitiera también analizar las dimensiones de la trasmisión integeneracional de las ideas, valores sociales y patrones culturales acerca de la anticoncepción adolescente. Situación que desafortunadamente está lejos de los alcances del presente estudio por no contar con información cualitativa al respecto. No obstante, este estudio aspira a contribuir al conocimiento de este fenómeno desde una óptica socio-demográfica basada en una metodológica cuantitativa.

\section{DESCENSO DE LA FECUNDIDAD, EMBARAZO Y ANTICONCEPCIÓN ADOLESCENTES EN MÉXICO}

La investigación demográfica da cuenta del notable descenso de la fecundidad ocurrido en el país durante las últimas cuatro décadas y la contribución que en ello han tenido los descensos observados en las tasas específicas de fecundidad de todos y cada uno de los grupos etáreos que componen a la población femenina en edad reproductiva: 15-49 años (Welti, 2005). Este tipo de estudios, sin embargo, también han señalado el comparativamente menor descenso observado en la tasa de fecundidad del grupo de la población adolescente (15-19). Asimismo, subrayan el papel estratégico que al respecto ha tenido el uso masivo de anticonceptivos sobre todo entre la población alguna vez unida de distintas edades, aunque también señalan que el uso ha sido menor entre las adolescentes de 15 a 19 años de edad (Welti, 2005) y durante la primera relación sexual (Gayet, 2014). 
En investigaciones recientes sobre el mismo tema se señala que si bien la fecundidad continúa descendiendo en el país, se observa un ritmo menor en el descenso al analizar la fecundad acumulada entre generaciones de mujeres alguna vez unidas que viven en áreas urbanas del país (Zavala y Páez, 2013). En concreto, las autoras plantean que el descenso de la fecundidad es comparativamente menor en la generación más joven de este tipo de mujeres respecto del descenso entre sus iguales pertenecientes a las dos generaciones inmediatas anteriores; así como también en lo que se refiere a su práctica anticonceptiva. Situación que en palabras de las autoras "puede estar relacionado con la menor reducción de la fecundad entre las generaciones más jóvenes" (Zavala y Páez, 2013: 14).

Lo anterior nos lleva a cuestionarnos acerca del por qué en un régimen de fecundidad controlada, como el que prevalece hoy en México, las mujeres unidas y urbanas de la generación más joven continúan iniciándose en la maternidad a edades muy tempranas al igual que antes lo hicieron sus madres y abuelas, pero en condiciones sociales de mayor vulnerabilidad social que en el pasado. Actualmente, las madres adolescentes tienen que enfrentar circunstancias muy distintas a las que, en su momento, enfrentaron sus iguales en tiempos pasados tanto en el ámbito familiar como en otras áreas de la vida social, pero en especial en las de la educación y el trabajo. Para tratar de entender tal situación es importante considerar qué está sucediendo con la práctica anticonceptiva de la actual población adolescente. Y para ello habría que examinar los posibles obstáculos a los que se enfrentan en los distintos ámbitos de su entorno social.

\section{Embarazo adolescente: un problema de salud y social en el México actual}

El embarazo entre las adolescentes continúa siendo un fenómeno que afecta a la sociedad mexicana y que preocupa a distintos sectores de la misma. Este es el caso de la comunidad científica en el área de la salud que lo identifica como un problema de salud pública que necesita solución (Soto et al., 2014). La investigación al respecto señala el impacto negativo que potencialmente tiene el embarazo a edades tempranas tanto sobre la salud de las madres adolescentes y de sus recién nacidos como, en un nivel extremo, en el aumento de los niveles de la mortalidad materna y de la mortalidad infantil, respectivamente (Soto et al., 2014; Welti, 2005). Asimismo, investigaciones en salud pública y en ciencias sociales han mostrado que este tipo de problemática actualmente afecta de manera principal a los grupos de población más desfavorecidos social y económicamente; convirtiéndolo 
Apertura hacia la anticoncepción adolescente entre los padres y las madres en México: un estudio de caso ... / N. OJEDA et al.

también en un problema de carácter social en varios países incluido México (Chandra-Mouli et al., 2014; Welti, 2005, Menkes y Suárez, 2013). Hoy en día, el embarazo adolescente está asociado a condiciones de desigualdad social en la población, lo que contribuye potencialmente a la perpetuación de tales condiciones en la sociedad en su conjunto, pero en especial entre las madres adolescentes y sus familias de procreación en el México actual.

Este doble problema de salud y social aún está lejos de ser superado en amplios sectores sociales de la población mexicana, debido a que se trata de un fenómeno que, por un lado, ha mostrado ser mucho más complejo de lo que la comunidad científica ha podido captar y, por otro, es más resistente de lo esperado al poder de intervención de las políticas públicas diseñadas hasta el día de hoy en el país. Especialistas en los temas de la sexualidad y el embarazo adolescente reconocen avances realizados al respecto. Esto es como resultado tanto de los programas de intervención creados y puestos en marcha por parte del estado mexicano, como por las organizaciones no-gubernamentales enfocados en la salud sexual y reproductiva de los adolescentes y jóvenes en el país. Algunos especialistas, sin embargo, también plantean la urgente necesidad de ampliar la comprensión de estos temas y la adopción de nuevos modelos para su evaluación e implementación más eficiente (Juárez y Gayet, 2005). Este es un aspecto muy relevante frente a la discusión entre algunos estudiosos acerca de si el embarazo adolescente es deseado o no. Al respecto, se argumenta que con cierta frecuencia las adolescentes de los grupos socio-económicos más desprotegidos ven en la maternidad adolescente una alternativa de participación social y de ascenso social simbólico en la escala socio-cultural de la sociedad mexicana. Esto es frente a sus muy limitadas oportunidades de desarrollo personal y otras formas de participación y ascenso social en el país (Stern y García, 2001; Menkes et al., 2015; Menkes y Suarez, 2013).

\section{Acerca de la anticoncepción adolescente}

Los estudios sobre la sexualidad y sobre la anticoncepción entre la población adolescente en México están basados en el uso de muy diversas fuentes de información, principalmente encuestas especializadas, con coberturas de población muy distintas y que utilizan diversas metodologías y definiciones de conceptos clave como son: adolescentes, jóvenes y sexualidad. Asimismo, los expertos señalan dificultades para realizar estudios comparativos con el rigor científico necesario hasta muy recientemente sobre estos temas debido a la falta de comparabilidad de los conceptos, de las fuentes de información y la ausencia de información confiable sobre estos 
temas entre generaciones más viejas. Si bien estos factores también dificultan poder tener cifras confiables y representativas a nivel nacional y sobre todo a nivel regional, a continuación se presentan algunos datos extraídos de un par de estudios basados en información proveniente de encuestas nacionales únicamente. Se busca resaltar algunos de los pocos aspectos que pueden ser generalizables a la población adolescente mexicana respecto de estos dos complejos temas.

Un primer aspecto se refiere a la intensidad de la práctica sexual entre los y las jóvenes en los inicios del presente milenio en México. Sobre esto, Welti (2005) aclara algunas aparentes contradicciones entre la impresión y rumor populares que indican la existencia de un aumento desmedido en la iniciación sexual de los y las jóvenes, por una parte, y por otra, la evidencia científica que en ese entonces se tenía al respecto. Con información de la Encuesta de Salud Reproductiva 2003 (ENSAR), el autor refuta tal impresión popular e incluso señala la presencia de una tendencia contraria que indica un retraso en las edades a la primera relación sexual y al nacimiento del primer hijo entre las mujeres alguna vez unidas de generaciones más jóvenes. Esta tendencia entra en aparente contradicción con la impresión popular arriba mencionada según el mismo autor por darse en un contexto social y demográfico que se caracteriza por un lado, por la existencia de un contingente de población comparativamente más numeroso de personas menores de los 20 años de edad y, por otro, por vivir en un ambiente social de mayor tolerancia y visibilidad de la sexualidad, especialmente entre los jóvenes; así como en un ambiente cultural cambiante acerca de lo que se entiende por ser joven versus ser adulto. Esto último como consecuencia, entre otros factores, de la presencia de duraciones más largas en las distintas etapas de la vida, producto del aumento en la esperanza de vida de los mexicanos. Asimismo, Welti (2005) identifica como factor explicativo de tal tendencia el aumento de la escolaridad entre las generaciones más jóvenes y el efecto diferencial directo de esta variable sobre la iniciación a la vida sexual y el nacimiento del primer hijo, donde se observa que a mayor escolaridad mayor son las edades a las que ocurren cada uno de estos eventos. Situación que, al parecer, hasta muy recientemente no había cambiado de manera notable. Al respecto, Gayet (2014) un estudio más reciente y utilizando encuestas de representación nacional señala que existe poco cambio en la edad de inicio sexual entre las generaciones más jóvenes.

Un segundo aspecto por considerar es la práctica anticonceptiva de los jóvenes. Los datos provenientes de varios estudios en lo general apuntan hacia una tendencia de mayor uso de anticonceptivos entre las mujeres 
Apertura hacia la anticoncepción adolescente entre los padres y las madres en México: un estudio de caso ... / N. OJEDA et al.

de generaciones más jóvenes. Nuevamente la ENSAR, 2003, proporciona información que permite identificar una tendencia creciente al respecto entre generaciones de mujeres alguna vez unidas en los inicios del presente milenio. Al respecto, Welti (2005) escribe

... aproximadamente 12 por ciento del total de las mujeres que tuvieron su primera relación sexual antes de 20 años usaron anticonceptivos, sin embargo, los porcentajes de usuarias muestran incrementos importantes en cada una de las generaciones sucesivas; de tal manera que entre las que tuvieron su primera relación entre los 16 y 19 años y pertenecen a la generación nacida entre 1979 y 1983 una de cada cinco mujeres usó anticonceptivos... entre la generación que nació... entre 1959 y 1963, solo una de cada dieciséis mujeres usó anticonceptivos (Welti, 2005: 156-157).

La tendencia señalada por Welti es sin duda un dato alentador, pero no así los niveles de uso de anticonceptivos que se observan por ser aún muy bajos incluso para la generación más joven incluida en esa encuesta levantada en el año 2003.

Información más reciente revela niveles más altos de uso de anticonceptivos entre la población femenina adolescente, pero también el que continua habiendo problemas en el uso y la eficiencia de anticonceptivos entre los jóvenes. Al respecto Rojas y Castrejón (2018) señalan el peso de la cultura de género que continúa restringiendo a las jóvenes, sobre todo de algunos grupos sociales, en el uso de anticonceptivos durante la iniciación de su vida sexual; esto es como parte de un "doble patrón moral" que aún prevalece respecto de la práctica sexual de ellos y ellas en el país. De tal modo que, si bien la tendencia de la tasa del embarazo adolescente en el país es hacia la baja, esta última continúa siendo relativamente alta. Al respecto, Menkes y Suárez señalan que:

el embarazo de mujeres de 15 a 19 años de edad ha descendido de manera gradual en las últimas décadas, aunque se observa un ligero repunte en 2008 ... sin embargo... la tasa de embarazo adolescente... en relación con las tasas de todas las mujeres de 15 a 49 años sigue siendo relativamente elevada, ya que representa hoy en día 15.2 por ciento de las tasas de embarazo total (Menkes y Suárez, 2013: 22).

Por su parte, investigadores del Instituto Nacional de Perinatología señalan que, en México

la Encuesta Nacional de Salud y Nutrición 2012 reportó un incremento del porcentaje de adolescentes que iniciaron vida sexual: 23 por ciento comparado 
con 15 por ciento del 2006. (Y)... el conocimiento sobre métodos anticonceptivos se ha incrementado... pues 90 por ciento reportó tener conocimiento de algún método... (los) adolescentes que iniciaron vida sexual sin protección disminuyó de 79.1 en 2000, en las mujeres a 33.4 por ciento en 2012 y en hombres de 47.5 a 14.4 por ciento, respectivamente ... A pesar del uso inicial de algún método anticonceptivo del total de las adolescentes que tuvieron relaciones sexuales 51.9 por ciento reportó haber estado alguna vez embarazada (Soto et al., 2014: 491).

\section{Estos mismos autores indican que}

...18.4 por ciento, es decir, prácticamente uno de cada cinco nacidos vivos en el 2011 son hijos e hijas de madres adolescentes. La tasa de fecundidad en 2011, de las mujeres entre 12 y 19 años, fue de 37 nacimientos por cada 1000 mujeres y de éstas hasta 40 por ciento no planearon ni desearon el embarazo (Soto et al., 2014: 491).

Lo anterior nos lleva a considerar otro aspecto problemático acerca de la práctica anticonceptiva entre los adolescentes. Varios estudios han demostrado que el conocimiento de métodos anticonceptivos entre los adolescentes no necesariamente conlleva a un mayor nivel de uso de los mismos en esta población. Esto es por intervenir aspectos de carácter más subjetivos como son los motivos por los que se tienen relaciones sexuales y el tipo de pareja con quien se inician y mantienen las relaciones sexuales. Factores que hacen de la practica anticonceptiva y la ocurrencia del embarazo temas más complejos que lo que ocurre entre la población adulta (Welti, 2005; Cacique, 2011, Gayet, 2014). De tal suerte que, una de las pocas evidencias empírica generalizables sobre el tema es que la edad al inicio sexual y el uso de anticonceptivos, durante la primera relación sexual entre los adolescentes, están inversamente relacionados con el nivel de escolaridad de los mismos y el estrato socioeconómico del que provienen (Menkes y Suárez, 2013; Gayet, 2014).

Otro tipo de aspectos también considerados en la investigación sobre la anticoncepción adolescente se refiere a su dimensión psicosocial (Brown, 2010), particularmente acerca de la decisión de usar o no usar anticonceptivos durante las relaciones sexuales y el deseo de convertirse en madres a corta edad. Aspecto necesario de estudiar desde una perspectiva analítica distinta a lo que ocurre entre la población adulta. En este sentido, habría que considerar los costos/ganancias sociales que potencialmente implica, para una adolescente iniciarse en la maternidad en el contexto social del 
Apertura hacia la anticoncepción adolescente entre los padres y las madres en México: un estudio de caso ... / N. OJEDA et al.

México actual; esto es particularmente a lo que a la educación y el empleo se refieren.

Sabemos que hasta muy recientemente, la escuela y el empleo en la vida de las mujeres han sido comparativamente más restringidas que para los hombres en México, al igual de lo que ocurre en otras sociedades. En el pasado esta situación no solo era socialmente más aceptada sino incluso era considerada "normal" y, en algunos sectores sociales, bienvenida dadas las expectativas socioculturales y de género predominantes que se tenían acerca de las mujeres; esto es casarse y ser madre a una "edad no muy mayor". Asimismo, un gran porcentaje de madres adolescentes estaban unidas conyugalmente y las que no lo estaban muy probablemente estaban en vías de estarlo, ya sea mediante un matrimonio forzado o en una unión libre como etapa prematrimonial, o bien símil de un matrimonio estable. Esto es dada la probable intervención de los padres de los menores de edad involucrados, quienes en ese entonces tenían más poder para influir respecto de esa importante "decisión" en las vidas de sus hijos e hijas apoyados frecuentemente por la legislación mexicana sobre el matrimonio entre menores de edad (Ojeda, 2013).

La ocurrencia de un embarazo adolescente por lo general siempre ha significado que las oportunidades de las jóvenes se vuelvan aún más limitadas, especialmente en lo que a la educación se refiere, por propiciar casi siempre que las adolescentes tengan que suspender y/o truncar sus estudios para dedicarse a los cuidados maternales. Pero hoy en día también conlleva a la madre adolescente a tener que iniciarse de manera temprana en el empleo para asumir la responsabilidad de proveedora de sí misma y de su recién nacido. Situación que a su vez contribuye a limitar aún más el abanico de oportunidades de empleo para las madres adolescentes; esto es tanto por sus menores niveles de educación alcanzados, como por encontrarse muchas veces sin el apoyo económico y emocional de una pareja conyugal estable para ejercer su nuevo rol de madres.

Los efectos que sobre la educación y el empleo contrae la maternidad adolescente sigue dándose en el mismo sentido que en el pasado pero, hoy en día tales efectos generan de manera frecuente mayores niveles de desigualdad social en la vida de las jóvenes madres y sus hijos. Por un lado, las condiciones del empleo actual demandan mayores niveles de educación por parte de ambos sexos en una economía de mercado cada vez más competitiva y globalizada. Y por otro, el efecto "protector" de tipo económico que tenía el matrimonio o la unión libre estable para la madre adolescente ha disminuido considerablemente, e incluso desaparecido en 
algunos sectores de la población. Esto es frente a los cambios que se están dando en los procesos de formación de las familias de procreación entre las generaciones más jóvenes y las expectativas sociales que hoy en día se dan acerca de la mujer en sus múltiples roles sociales, como madre, trabajadora, proveedora del hogar y menos frecuentemente de esposa o compañera en una unión conyugal estable (Ojeda, 2013).

La importancia creciente de la educación y el empleo en las vidas de las mexicanas de todas edades, ya sean solteras o casadas, tengan hijos o no los tengan, hacen del embarazo adolescente un problema social aún más apremiante que requiere ser atendido de manera integral. Con esto queremos decir que es necesario seguir avanzando en su estudio considerando otro tipo de aspectos que lo contextualizan como fenómeno social; lo cual nos obliga a analizar los ámbitos sociales de interacción de los y las adolescentes como actores sociales. Asimismo, si bien el y/o la adolescente son los actores principales en esta problemática no son los únicos y sería necesario también considerar a otros actores sociales en dicha problemática y sus espacios. En este sentido, podemos pensar en el ámbito familiar es un espacio social privilegiado de observación y en los padres por ser actores sociales importantes dado su doble rol de agentes socializadores y agentes de supervisión y control social de los niños y de una buena parte de las vidas de los y las adolescentes que viven en el hogar paterno y/o materno. Esto es concretamente en lo que se refiere a la trasmisión inter-generacional de ideas, valores socioculturales, así como posibles prácticas acerca de la sexualidad y la anticoncepción por parte de los padres hacia los hijos e hijas durante la infancia y adolescencia. Es con este interés que el presente estudio tiene por objetivo analizar las actitudes o posturas de los padres de familia acerca de la sexualidad y la anticoncepción adolescentes. Esto es importante de ser estudiado debido al role privilegiado que tienen los padres y las madres en el proceso de socialización de los niños y adolescentes; así como el control social que ejercen sobre los mismos especialmente en el espacio social familiar y dada la legitimidad social de la que gozan al respecto en la sociedad mexicana, al igual de lo que ocurre en la mayoría de las sociedades. Se parte del supuesto teórico analítico de que dichas actitudes o posturas reflejan, de manera directa e indirecta, las ideas, los valores sociales y de género-culturales, así como prácticas en materia de sexualidad y la anticoncepción que son trasmitidas de padres y madres a hijos e hijas, mediante el proceso de socialización de los niños y adolescentes en el seno familiar. 
Apertura hacia la anticoncepción adolescente entre los padres y las madres en México: un estudio de caso ... / N. OJEDA et al.

\section{Familia y socialización de niños y adolescentes respecto de la sexualidad y el género}

El tema de la socialización es sin duda una las contribuciones más importantes de la sociología para estudiar los marcos sociales y culturales que regulan la trasmisión de las ideas, los valores sociales, culturales y los códigos del comportamiento social entre las generaciones. Se trata de un proceso harto complejo el cual es llevado a cabo por varias instancias, agentes e instituciones sociales del que, en última instancia, depende gran parte la reproducción social de los pueblos. Al respecto, Ojeda y González (2019: 174-176) en un estudio relacionado con un tema afín al que aquí nos ocupa, citan algunos componentes de la socialización, apoyándose en contribuciones realizados por otros autores sobre el tema en lo general y en particular con el papel o role de la familia en la socialización de los menores de edad. Así, Ojeda y González (2019) citando a Elkin y Handel ${ }^{1}$ señalan que

el proceso de socialización es aquel mediante el cual uno aprende las maneras de actuar en una sociedad dada o de un grupo social dado y que permite que uno funcione en dicha sociedad o grupo. Se trata de un proceso cognitivo consciente que nos remite al cómo uno aprende a adoptar actitudes y conductas consideradas por la sociedad como culturalmente apropiadas para uno, según nuestra edad y sexo, entre otras características, y en anticipación a lo que socialmente se espera de nosotros en el corto y largo plazo (Ojeda y González, 2019: 175).

Asimismo, Ojeda y González (2019) nos recuerdan que la socialización permite la adaptación social de las personas conforme a los convencionalismos vigentes en tiempo y espacio de cada sociedad, señalando que su importancia vaya más allá de la adaptación social de los individuos ya que "de la socialización mucho depende que se dé la reproducción social de las sociedades y sus estructuras sociales, incluyendo los sistemas de sexo-genero de cada sociedad y época histórica" (Ojeda y González, 2019: 176).

Son varios los espacios sociales y las instituciones encargadas directa e indirectamente de la socialización de los miembros de las nuevas generaciones. Entre estas destaca la familia por ser en ésta donde se inicia el proceso y se fijan los primeros rasgos de la personalidad en los infantes y adolescentes. Sobre este aspecto, Ojeda y González retoman a Inkeles $(1968)^{2}$ y citan su definición de socialización como:

\footnotetext{
${ }^{1}$ Elkin y Handel (1984: 33-76).

${ }^{2}$ Inkeles (1968).
} 
como... el proceso mediante el cual una persona adquiere las actitudes, valores, maneras de pensar, inclinaciones y otros atributos personales, aunque sociales, que la van a caracterizar en la siguiente fase de su desarrollo... En la interacción con el niño, los padres y otros agentes socializadores generalmente tienen en mente cierta concepción acerca de lo que el niño supuestamente se convertirá y del papel que una determinada practica de crianza podrá tener en alcanzar o bien obstaculizar el producto final que es deseable (Ojeda y González, 2019: 175-176).

Con el interés de profundizar acerca de la socialización de los menores en el espacio familiar, Ojeda y González (2019) basados en otros estudios, señalan que como parte del mismo proceso, los niños y las niñas aprenden mediante la observación de las personas que los rodean conductas de ambos sexos, pero pronto también aprenden que ellos serán premiados por un tipo de conducta pero no por la otra y como esto se va a dejar sentir en las distintas etapas de la vida de las personas. Sobre este aspecto y aplicándolo a la socialización de los y las menores de edad, en materia de igualdad de género, Ojeda y González (2019: 175) retoman a Marini y Fan ${ }^{3}$ quienes, a su vez, plantean que "la influencia formativa que tienen las actitudes y conductas de los padres sobre la mayor o menor apertura en pro de la equidad de género de sus hijos e hijas no solo durante la niñez, sino también y de manera importante durante las edades adultas". Aspecto que nos permite ampliar nuestro entender acerca de la importancia de la socialización de los niños y adolescentes en el espacio familiar no solo durante la convivencia de padres e hijos cuando estos son menores de edad, sino a posteriori en la vida adulta de las personas.

Finalmente, podríamos decir que la familia constituye tal vez el espacio más importante en el proceso de socialización de los niños y adolescentes en México porque, a diferencia de otros posibles espacios de socialización a edades tempranas, la familia ejerce un periodo de influencia notablemente más largo que, por ejemplo, la escuela. Esto es ya sea porque hay niños que no pueden acudir a la escuela o bien lo hacen por solo poco tiempo al tener que dejar de estudiar muchos niños que por necesidad o costumbre comienzan a trabajar a corta edad. Esto es sin afán de minimizar la importancia de la escuela en la socialización de los niños y adolescentes en el país.

\section{EI caso de Tijuana, Baja California}

El comportamiento diferencial del embarazo adolescente, según el estrato socioeconómico al que pertenecen los adolescentes, ha sido planteado con-

\footnotetext{
${ }^{3}$ Marini y Fan (2000).
} 
Apertura hacia la anticoncepción adolescente entre los padres y las madres en México: un estudio de caso ... / N. OJEDA et al.

forme a la evidencia empírica que proporcionan las investigaciones sobre el tema. La complejidad del comportamiento de este fenómeno, sin embargo, hace difícil hacer generalizaciones sobre el mismo. Un ejemplo de esta situación se presenta en la aparente contradicción del caso del embarazo adolescente en el estado de Baja California de manera reciente presentara uno de los niveles más altos a pesar de ser un estado con un grado de desarrollo económico y social más altos en el país. De tal modo que, como señalan Palma y Redign (2011: 81) para el año 2005, el estado de Baja California mostraba la segunda tasa de fecundidad más alta para el grupo 1519 (después del estado de Chiapas) en contraste con este estado que tiene uno de los niveles de desarrollo más bajos. Palma (2019: 8) señala que para el año 2006, la tasa de fecundidad adolescente de Baja California había descendido aunque aún colocaba al estado por arriba del nivel nacional y entre los seis estados con los niveles de fecundidad adolescente más altos en México. Esta misma autora calcula la tasa de fecundidad de las jóvenes de 15-19 años de edad en 77.3 para Baja California y en 69.2 para el país en su conjunto, utilizando información de la Encuesta Nacional Demográfica, 2009 (Palma, 2019: 22).

Las variaciones regionales sobre el tema y sus explicaciones no son tan directas. En el caso de Baja California desafortunadamente la investigación científica al respecto es limitada, pero entre las pocas contribuciones a la explicación de tal aparente contradicción se mencionan los posibles efectos variados que pudieran derivarse del fenómeno migratorio en el estado y el acelerado crecimiento demográfico (Palma, 2019; Palma y Reding, 2011). Al respecto, importa mencionar que la inmigración al estado y particularmente a Tijuana ha sido muy elevada por largo tiempo, sino también, ha sido muy compleja socialmente, entre otras razones, por los múltiples lugares de origen (y por las mismas subculturas) de sus inmigrantes. Esto es además de las complejidades culturales y sociales que se derivan de su vecindad geográfica con el estado de California del país vecino del norte y los procesos de difusión cultural que se dan entre ambos países, pero en especial entre sus jóvenes.

Asimismo, estudios sobre la sexualidad y reproducción de los jóvenes en Tijuana señalan, por un lado, una iniciación sexual más precoz entre las jóvenes inmigrantes que entre las nacidas en cualquiera de los municipios del estado incluido Tijuana (Palma y Reding, 2011). Y por otro, que la incidencia de las infecciones de transmisión sexual ha afectado a casi la décima parte de los habitantes de Tijuana de edades 15-19 años; al tiempo que la mayor parte de la población de edades 15-29 años de edad que 
han experimentado algún padecimiento de este tipo (53.4 por ciento), fueron contagiados cuando eran aún adolescentes (González, 2011: 29). Este último autor también presenta información sobre proporciones más altas de embarazos durante la adolescencia entre las jóvenes inmigrantes que entre las jóvenes nacidas en el estado (González, 2011: 30-31).

Entre los múltiples factores que pudieran estar contribuyendo a esta problemática habría que considerar algunos referidos al contexto social donde se desenvuelven los adolescentes de Tijuana. Concretamente, el impacto directo e indirecto que pudieran estar teniendo las políticas públicas estatales relacionadas con la salud sexual y reproductiva de los adolescentes, tanto en sector salud, como en el educativo. Respecto al primero, se tienen los cambios hechos a la ley sobre el aborto, por parte del sector más conservador de la elite política de Baja California en el año 2008. La nueva ley criminaliza el aborto en todas las circunstancias habiendo eliminado las tres causales que antes lo permitían durante los tres primeros meses de la gestación (Aguilar, 2014). Esto se dio en un tiempo en el que un alto porcentaje de la población femenina del estado apoyaba la idea de que las mujeres deberían poder abortar legalmente cuando la salud de la mujer está en peligro, cuando hay evidencia de defectos congénitos en el bebé, y cuando el embarazo es resultado de violación (Ojeda, 2001: 54). Este estudio también detectó que 78 por ciento de las mujeres entrevistadas opinaban que "la información (sobre los diferentes métodos anticonceptivos) debe estar disponible para los adolescentes" (Ojeda, 2001: 52).

En el ámbito educativo, se tiene la circunstancia de que los estudiantes de secundarias públicas no cuentan con programas de educación sexual en sus escuelas y cuando reciben algún tipo de información al respecto, ésta frecuentemente es de dudosa calidad, entre otras cosas, por manejar mensajes inconsistentes entre sí y que, frecuentemente, se contradicen con algunas prácticas y actitudes al respecto por parte de los mismos docentes que proporcionan tal información (Islas, 2017). Asimismo, otros estudios han encontrado que el ámbito familiar bajacaliforniano no necesariamente proporciona a los niños y adolescentes acceso a información oportuna y de calidad sobre la sexualidad y la anticoncepción (Ramos, 2016; Aguilar et al., 2019). Es en este contexto en el que los padres y madres de Baja California se dan a la tarea de socializar a los niños y adolescentes en las áreas de salud sexual y reproductiva, incluyendo la anticoncepción. 
Apertura hacia la anticoncepción adolescente entre los padres y las madres en México: un estudio de caso ... / N. OJEDA et al.

\section{Metodología y fuente de datos}

Se analizan las posturas de padres y madres de familia con hijos e hijas en edades de educación secundaria acerca de informar sobre la sexualidad y el uso de anticonceptivos a los adolescentes de ambos sexos. Para tal propósito se utiliza información proveniente de la "Encuesta a Padres de Familia con Hijos en Escuelas Secundarias, B.C." Se trata de una encuesta de conveniencia llevada a cabo con las madres y los padres de los alumnos de cuatro escuelas secundarias públicas en el municipio de Tijuana durante la primavera del año 2015. La localización de las escuelas en el municipio y el tipo de escuelas encuestadas (una técnica y tres tradicionales) nos permite contar con un universo de padres de familia de ambos sexos, cuya clase social corresponde a los sectores de la clases media trabajadora popular y obrera que radican en Tijuana. De modo que, en su mayoría se trata de padres con ocupaciones tales como empleados del comercio establecido a menudeo, empleados de bajo rango en oficinas públicas, maestros de primarias y secundarias públicas, obreros de la industria maquiladora, vendedores ambulantes y trabajadores por su cuenta. También algunos indicaron no trabajar y/o estar desempleados al momento de la encuesta.

El método del levantamiento de la información consistió en aplicar un cuestionario estandarizado con preguntas cerradas que fue auto administrado por los mismos padres y madres durante las reuniones de padres de familia en los planteles educativos. Estas últimas fueron organizadas por las escuelas con el fin de entregar las boletas de calificaciones de los alumnos a mediados del ciclo escolar. En total se encuestaron a 2,815 adultos, de los cuales 2,547 (90.5 por ciento) son madres o padres de los y las alumnas, y son precisamente éstos quienes son incluidos en este estudio, independientemente del tipo de arreglo domiciliario que tenían al momento de la encuesta.

La información así obtenida se analiza a partir de la obtención de frecuencias y estadísticas descriptivas de las distintas variables sociales y demográficas consideradas, así como de los varios reactivos aplicados a la población de padres y madres acerca de la sexualidad y la anticoncepción entre los adolescentes. Asimismo, se diseñó un "índice de apertura" para medir el nivel de acuerdo de ellos y ellas a proporcionar a los adolescentes información acerca de la sexualidad y la anticoncepción en general y con equidad de género. El índice se construyó a partir de los reactivos que a continuación se mencionan; otorgando un punto por cada postura a favor de los reactivos que denotan apertura, y un punto por cada postura 
en contra de los reactivos que infieren condena hacia la equidad de género en la anticoncepción adolescente y acerca de la sexualidad de los y las adolescentes. Con base en este índice se clasifican a los padres y las madres respecto de su nivel de apertura en las dos áreas. De modo que un puntaje de seis o menos implica una "actitud cerrada", de siete a nueve denota una "actitud semi-abierta", y un puntaje de 10, 11 ó 12 (máximo) una "actitud abierta" respecto de la sexualidad y la anticoncepción adolescente en general y con equidad de género.

A saber, los reactivos que se consideran denotar una postura en "favor" en las dos áreas consideradas, son los siguientes:

1. Actualmente ya existe igualdad entre hombres y mujeres.

2. La edad para permitir el noviazgo puede ser la misma tanto para las hijas como para los hijos.

3. La educación sexual se debe ofrecer por igual a las hijas que a los hijos.

4. Mamá y papá están mejor preparados que los maestros para educar a los hijos e hijas acerca de la sexualidad.

5. Los padres deberían hablar sobre sexo y anticonceptivos a sus hijos e hijas, antes de los 13 años de edad.

6. Informar a los hijos e hijas sobre métodos anticonceptivos es una obligación de mamá y papá.

7. Para evitar el embarazo adolescente los padres (de ambos sexos) deben proporcionar y enseñar a usar anticonceptivos a sus hijas.

Y los reactivos que se consideraron denotar condena acerca de las mismas dos areas en lo general y con equidad de género, son las siguientes:

1. En cuanto a los permisos para salir las reglas deben ser más estrictas para las hijas que para los hijos.

2. La educación sexual de los hijos e hijas debe darse en la escuela en lugar de en la casa.

3. Hablar sobre sexo a los hijos es como darles permiso para que ellos tengan relaciones sexuales.

4. Los padres que hablan sobre sexo a sus hijos podrían perder su respeto.

5. No es necesario usar el condón para que los hijos se protejan de contraer enfermedades de transmisión sexual.

Finalmente, se estiman algunos modelos estadísticos de tipo logísticos para identificar los factores sociales y demográficos que favorecen una actitud o postura abierta por parte de los padres y las madres hacia la anticoncepción adolescente. Para esto se consideran algunas variables que han 
Apertura hacia la anticoncepción adolescente entre los padres y las madres en México: un estudio de caso ... / N. OJEDA et al.

demostrado ser significativas en otros estudios sobre el tema. Se trata de las variables sexo, condición laboral, afiliación religiosa, condición migrante y tiempo de vivir en Tijuana, así como la existencia de un embarazo y/o maternidad adolescente en la historia reproductiva de las madres y de las esposas o compañeras conyugales de los padres varones.

\section{Resultados}

En un primer nivel de análisis se identifico el perfil sociodemográfico de la población encuestada y que se presenta en la Tabla 1. Como puede observarse, en su mayoría se trata de madres que acudieron a recoger las boletas de calificaciones de sus hijos e hijas representando 81.7 por ciento de los casos; mientras que los padres solo representan 18.3 por ciento del total de la población encuestada. Este es un dato importante a considerar dado el posible sesgo que pudiera introducir al momento de analizar los resultados que a continuación se presentan y las posibles conclusiones que de ello se deriven.

Tabla 1: Perfil sociodemográfico de los padres de familia encuestados. Tijuana, Baja California, 2015

\begin{tabular}{lr}
\hline Sexo (\%) & Indicador \\
\hline Femenino & 81.7 \\
Masculino & 18.3 \\
Edad (media \pm DE) & $40.1 \pm 6.6$ \\
Población no nacida en la entidad (\%) & 64.1 \\
Tiempo de residir en Tijuana (media \pm DE) & $20.6 \pm 10.4$ \\
Tiene un empleo remunerado (\%) & 69.2 \\
Declararon tener alguna religión (\%) & 83.3 \\
\hline * $<0.1 \quad$ ** $<0.05 \quad$ *** $<0.01$ & \\
Fuente: Estimaciones propias con base en la Encuesta a Padres de Familia con Hijos en Escuelas \\
Secundarias-Tijuana, Baja California.
\end{tabular}

Respecto de la edad, y considerando de manera conjunta a los dos sexos, tenemos que su edad promedio es de 40.1 años. Este promedio indica que se trata de una población de padres y madres jóvenes y de edades intermedias por tratarse, en un gran número de mamas y papás con hijos en edades propias de escuela secundaria e incluso con hijos aún más pequeños. Nótese también que la mayoría de ellos y ellas declararon tener algún tipo de religión, con 83.3 por ciento de casos, siendo principalmente de 
tipo católica. En cuanto a su condición migratoria, podemos ver que una muy alta proporción de ellos y ellas son inmigrantes, ya que 64 por ciento declararon haber nacido en un estado distinto al de Baja California; mismos que, al parecer, ya tienen cierto arraigo en el estado por tener en promedio 20.6 años de vivir en Tijuana. Finalmente, 69.2 por ciento de esta población declaro tener un empleo. Esta cifra significa que, en contraparte, cerca de una tercera parte (30.8 por ciento) no trabajan o estaban desempleados al momento de la encuesta. Esta situación que pudiera deberse al hecho de tratarse de una población primordialmente compuesta por madres, como se hizo notar antes, y que por lo mismo se declaran como amas de casa.

Tabla 2: Niveles de acuerdo, entre la población total de padres y madres, acerca de la sexualidad y la anticoncepción adolescentes y con equidad de género, Tijuana, Baja California 2015

\begin{tabular}{lr}
\hline Reactivo/Enunciado & $\begin{array}{r}\text { De acuerdo con el } \\
\text { enunciado (\%) }\end{array}$ \\
\hline $\begin{array}{l}\text { 1. Actualmente ya existe igualdad entre hombres y mujeres } \\
\text { 2. En cuanto a los permisos para salir, las reglas deben }\end{array}$ & 74.4 \\
ser más estrictas para las hijas que para los hijos & 21.3 \\
$\begin{array}{l}\text { 3. La edad para permitir el noviazgo puede ser la misma } \\
\text { tanto para las hijas como para los hijos }\end{array}$ & 80.2 \\
$\begin{array}{l}\text { 4. La educación sexual se debe ofrecer por igual a las hijas } \\
\text { que a los hijos }\end{array}$ & 94.3 \\
$\begin{array}{l}\text { 5. La educación sexual de los hijos e hijas debe darse en la escuela } \\
\text { en lugar de en la casa }\end{array}$ & 14.0 \\
$\begin{array}{l}\text { 6. Mamá y papá están mejor preparados que los maestros } \\
\text { para educar a los hijos acerca de la sexualidad }\end{array}$ & 39.7 \\
$\begin{array}{l}\text { 7. Los padres deberían hablar sobre sexo y anticonceptivos } \\
\text { a sus hijos e hijas antes de los 13 años de edad }\end{array}$ & 84.7 \\
$\begin{array}{l}\text { 8. Hablar sobre sexo a los hijos es como darles permiso } \\
\text { para que ellos tengan relaciones sexuales } \\
\text { 9. Los padres que hablan sobre sexo a sus hijos podrían perder } \\
\text { su respeto }\end{array}$ & 4.9 \\
$\begin{array}{l}\text { 10. Informar a los hijos e hijas sobre métodos anticonceptivos } \\
\text { es una obligación de mamá y papá }\end{array}$ & 5.1 \\
$\begin{array}{l}\text { 11. No es necesario usar el condón para que los hijos se protejan } \\
\text { de contraer enfermedades de transmisión sexual } \\
\text { 12. Para evitar el embarazo adolescente los padres deben } \\
\text { proporcionar y enseñar a usar anticonceptivos a sus hijas }\end{array}$ & 88.9 \\
\hline Fut Es & 13.4
\end{tabular}

Fuente: Estimaciones propias con base en la Encuesta a Padres de Familia con Hijos en Escuelas Secundarias-Tijuana, Baja California. 
Sobre las actitudes o posturas de la población encuestada respecto de la sexualidad y la anticoncepción adolescentes, primero se presentan los resultados obtenidos para el total de padres de ambos sexos en cada uno de los reactivos considerados. Así, en la Tabla 2 podemos observar que en la mayoría de los reactivos o enunciados considerados se dan altos porcentajes, ya sea de aceptación o bien de rechazo y son pocos los reactivos en los que se evidencian actitudes o posturas dividas entre la población total de padres de ambos sexos.

Un primer dato que llama la atención es que la mayoría declaró estar de acuerdo con el reactivo que plantea que 'actualmente ya existe igualdad entre hombres y mujeres' con 74.4 por ciento de los casos. Esta postura en lo general, coincide con los altos porcentajes que indican estar a favor de condiciones de equidad de género respecto a informar a los hijos e hijas acerca de la sexualidad y la anticoncepción, así como respecto a la disciplina. De tal modo que, prácticamente toda la población encuestada (94.3 por ciento) está de acuerdo en que 'la educación sexual se debe ofrecer por igual a las hijas que a los hijos'. También en el mismo sentido, casi la mayoría de la población total declaró no estar de acuerdo con los enunciados que indican que 'hablar sobre sexo a los hijos es como darles permiso para que ellos tengan relaciones sexuales' y que 'los padres que hablan sobre sexo a sus hijos podrían perder su respeto' ya que solo 4.9 y 5.1 por ciento, respectivamente apoyan tales ideas. Finalmente, en cuanto a la disciplina en casa, solo un poco más de la quinta parte de la población en estudio piensa que 'en cuanto a los permisos para salir, las reglas deben ser más estrictas para las hijas que para los hijos'; mientras 80 por ciento considera que 'la edad para permitir el noviazgo puede ser la misma para las hijas como para los hijos'.

Estos resultados en lo general indican una clara postura a favor de proporcionar información sobre la sexualidad y la anticoncepción y con equidad de género a los y las adolescentes. Sin embargo, tal postura se debilita al diferenciar a la población encuestada según su sexo, como se muestra más adelante.

Acerca de quién debe informar a los y las adolescentes sobre estos temas, nótese que la mayoría de la población bajo estudio parece estar de acuerdo en participar en la educación sexual de sus hijos e hijas. Sin embargo, esto se da en términos un tanto contradictorios. Por un lado, solo el 14 por ciento está de acuerdo con el enunciado de que 'la educación sexual de los hijos debe darse en la escuela en lugar de en la casa', pero, por otro lado, contradictoriamente solo poco menos de 40 por ciento está de acuer- 
do con la idea de que 'mamá y papá están mejor están mejor preparados que los maestros para educar a sus hijos acerca de la sexualidad'. Y en lo que a la anticoncepción adolescente se refiere, nótense los altos porcentajes de la población que están de acuerdo con los enunciados a favor de informar y facilitar el uso de anticonceptivos. Prácticamente 89 por ciento está de acuerdo con el enunciado de que 'informar a los hijos e hijas sobre métodos anticonceptivos es una obligación de mamá y papá', y casi tres de cada cuatro están a favor de proporcionar y enseñar a usar anticonceptivos a sus hijas para evitar el embarazo adolescente. Por su parte, solo 13 por ciento acepta la idea de que 'no es necesario usar el condón para que los hijos se protejan de contraer enfermedades de transmisión sexual'. Estos resultados, también se modifican al diferenciar entre las madres y los padres, como se verá más adelante.

El efecto diferencial de la variable sexo en el análisis de las actitudes puede verse en la Tabla 3, donde se presentan los resultados obtenidos para cada uno de los mismos enunciados considerados. En la mayoría de los casos no se presentan diferencias significativas entre las madres y los padres. No obstante, se puede ver una postura notablemente más a favor entre las madres que entre los padres a favor de informar acerca de la sexualidad y la anticoncepción a los adolescentes de ambos sexos. En cambio los padres están más a favor de que las reglas sean más estrictas para las hijas que para los hijos, en cuanto a los permisos para salir se refiere, y prefieren que la educación sexual se dé en la escuela en lugar de la casa. Asimismo, ellos son más proclives que ellas a tener una actitud o postura en contra del condón como protección de enfermedades de transmisión sexual; así como de proporcionar y enseñar a las hijas a usar anticonceptivos para evitar embarazos durante la adolescencia.

Grosso modo, estos datos indican actitudes o posturas diferentes entre los padres y las madres. De tal modo que, ellas tienen, en comparación con ellos, una más clara postura a favor tanto de informar acerca de la sexualidad y la anticoncepción adolescente; así como hacerlo con equidad de género.

Frente a este último resultado, se consideró conveniente analizar el impacto potencial que pudiera tener el trabajo remunerado entre las madres en sus posturas en estos mismos temas. Como puede verse en la misma Tabla 3, los resultados así obtenidos; sin embargo, no son los teóricamente esperados, ya que el hecho de trabajar a cambio de un salario por parte de las madres, al parecer, no imprime diferencias significativas entre ellas excepto en dos enunciados. 
Apertura hacia la anticoncepción adolescente entre los padres y las madres en México: un estudio de caso ... / N. OJEDA et al.

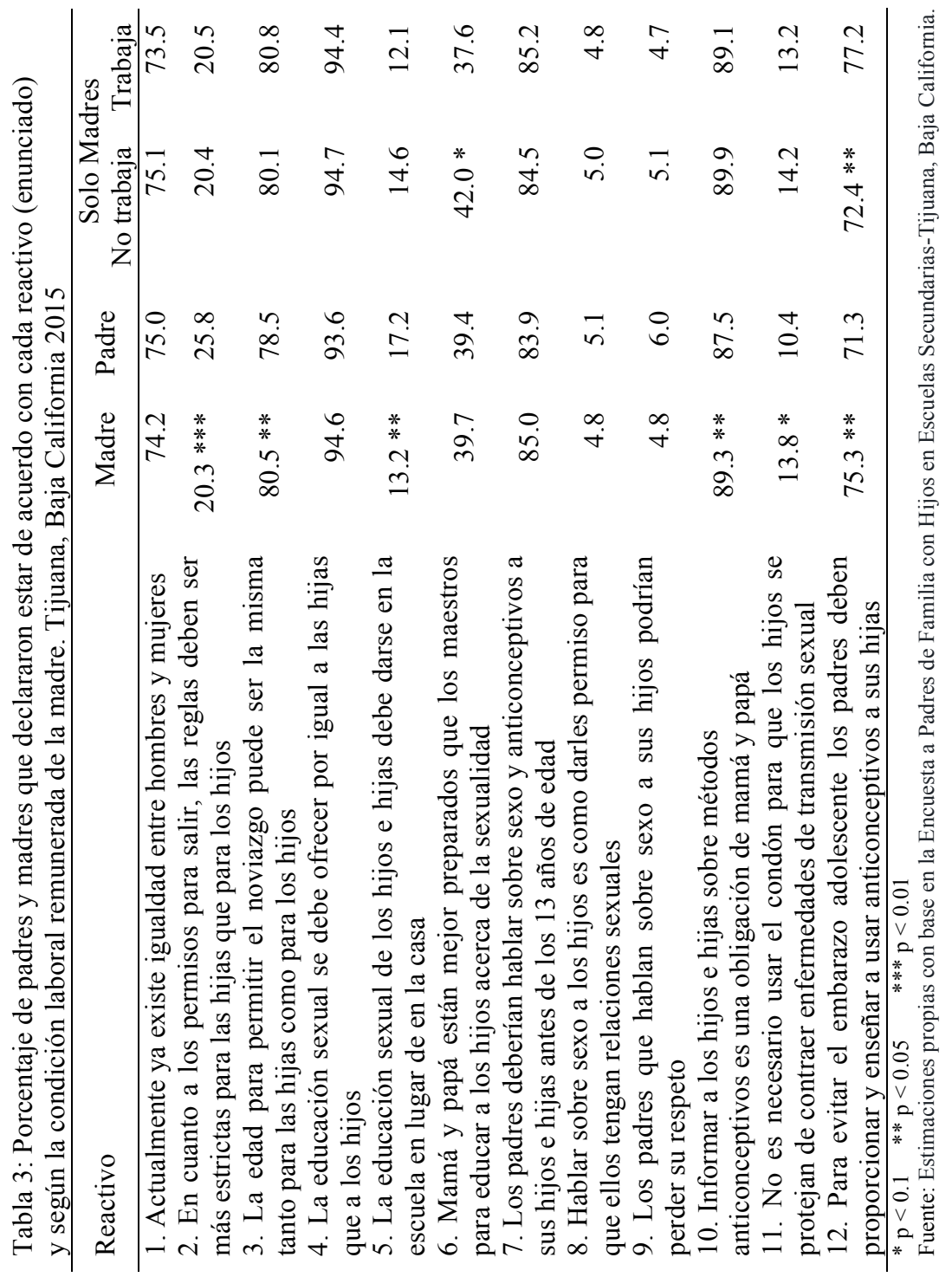


Interesantemente, las madres que no trabajan están más convencidas que las que si trabajan de que tanto mamá y papá están mejor preparados que los maestros para educar a los hijos de ambos sexos en materia de sexualidad; así como de que para evitar el embarazo adolescente los padres y las madres deben proporcionar y enseñar a las hijas a usar anticonceptivos.

Los hallazgos arriba descritos sin duda nos permiten tener una primera aproximación acerca de las posturas de los padres y las madres sobre los temas que aquí nos ocupan.

No obstante, se consideró la necesidad de profundizar al respecto reconociendo dos cosas importantes. Primero la necesidad de confirmar las diferencias entre las madres y los padres, y segundo, que las posturas no tienen necesariamente que ser dicotómicas ni excluyentes entre sí; esto es a favor o en contra en cada uno de los reactivos utilizados en el análisis y sus posibles combinaciones entre sí. Lejos de ello, se considera que existe un gradiente en las posturas que conviene medir para así acercarnos de mejor manera a la realidad en estos temas sensibles. Con este interés se procedió a diseñar un "índice de apertura" a favor de la sexualidad y la anticoncepción adolescentes con equidad de género que nos permite medir de manera resumida la postura general de los padres y las madres al respecto.

Los hallazgos así obtenidos se presentan en la Tabla 4. En este último podemos ver que se confirma de manera contundente la diferencia por sexo en la apertura hacia la sexualidad y la anticoncepción adolescentes y con equidad de género entre la población bajo estudio. Las madres tienen una mayor apertura al respecto que los padres varones, con valores en los índices de 60.9 versus 52.3 por ciento, respectivamente. Asimismo, nótese que el gradiente que se da en el índice de apertura entre las madres es más marcado que entre los padres varones. De tal modo que, la diferencia entre las madres que tienen una apertura abierta y aquellas con una apertura entreabierta es de casi 27 puntos porcentuales; en tanto que la diferencia respectiva entre los padres varones de solo 8.4 puntos. En otras palabras, las madres no solo tienen una mayor apertura que los padres varones sino también su apertura es más contundente.

Finalmente, se estimaron tres modelos de regresión logística, en donde el índice de apertura en favor de la sexualidad y la anticoncepción adolescente, reclasificada de manera dicotómica ("abierta y "no abierta") es la variable dependiente. El propósito de esta parte del análisis es identificar los factores que pudieran estar determinando los niveles de apertura de la población de padres de ambos sexos, según algunas de sus características sociodemográficas. 
Apertura hacia la anticoncepción adolescente entre los padres y las madres en México: un estudio de caso ... / N. OJEDA et al.

Tabla 4: Niveles de apertura en favor de la sexualidad y la anticoncepción adolescente entre padres de distinto sexo. Tijuana, Baja California 2015

\begin{tabular}{lcccc}
\hline Respondientes * & \multicolumn{3}{c}{$\begin{array}{c}\text { Apertura en favor de la sexualidad y la } \\
\text { anticoncepción adolescente }\end{array}$} & Total \\
\hline \multirow{3}{*}{ Madres } & Cerrada & Entreabierta & Abierta & \\
Padres & 4.9 & 34.2 & 60.9 & 100 \\
Total & 4.8 & 43.9 & 52.3 & 100 \\
\hline
\end{tabular}

$* \mathrm{p}<0.005$

Fuente: Estimaciones propias con base en la Encuesta a Padres de Familia con Hijos

en Escuelas Secundarias-Tijuana, Baja California.

En el primer modelo solo se incluye la variable sexo y los resultados así obtenidos se presentan en la Tabla 5. En este último es notoria la significancia estadística de esta variable por indicar que las madres son 42.3 por ciento más probables que los padres de tener una apertura abierta o favorable hacia la sexualidad y la anticoncepción adolescente con equidad de género.

En el segundo modelo se incluye además otras variables que son la escolaridad, el número de hijos nacidos vivos y los antecedentes de maternidad adolescente en la historia reproductiva de la madre o bien de la esposa o compañera conyugal del padre varón.

Sobre la educación, en la Tabla 5 observamos que los padres con una escolaridad mayor al nivel de secundaria tienen 16.5 por ciento más de probabilidad estar a favor y, por lo mismo, de tener apertura abierta hacia la sexualidad y anticoncepción adolescente. De manera similar, aquellas personas que experimentaron una maternidad adolescente directamente en caso de ser mujer, o indirectamente, en caso de ser hombre, tienen poco más de 21 por ciento más de probabilidad de tener una postura abierta hacia la sexualidad y la anticoncepción adolescente. En cambio una "fecundidad alta" (tres hijos o más) no parece favorecer la apertura en pro de la sexualidad y anticoncepción adolescente.

Por último, importa mencionar que el tercer modelo logístico se estimó solo para la población de madres, pero los resultados obtenidos, como pueden verse también en la Tabla 5 no indican diferencias importantes respecto del modelo anterior. Un resultado que en este sentido es importante de ser subrayado es el que se refiere a los antecedentes de maternidad adolescente entre las madres encuestadas. Al parecer esta característica está estadísticamente asociada a una mayor apertura tanto entre las madres, 
que experimentaron un embarazo adolescente en su historia reproductiva, como entre los padres varones que también lo experimentaron, pero de manera indirecta, vía sus esposas o compañeras conyugales (segundo modelo). En otras palabras, el haber vivido de manera directa o indirecta la maternidad adolescente genera una mayor apertura a favor de la equidad de género acerca de la sexualidad y la anticoncepción adolescentes entre las madres y los padres.

Tabla 5: Coeficientes de regresión logística para la Apertura hacia la anticoncepción entre los padres y madres de adolescentes. Tijuana, Baja California2015

\begin{tabular}{|c|c|c|c|}
\hline Factor & Modelo 1 & Modelo 2 & $\begin{array}{r}\text { Modelo } 3 \\
\text { (Solo madres) }\end{array}$ \\
\hline Constante & 1.095 & 1.009 & 1.356 \\
\hline $\begin{array}{l}\text { Sexo = mujer } \\
(\text { IC 95\%) }\end{array}$ & $1.423^{* * *}$ & $1.424^{* * *}$ & \\
\hline Ref $=$ hombre & $(1.162-1.743)$ & $(1.160-1.748)$ & \\
\hline $\begin{array}{l}\text { Fue madre adolescente } \\
\text { (IC 95\%) }\end{array}$ & & $1.212^{* *}$ & $1.21^{\star *}$ \\
\hline $\begin{array}{l}\text { Ref }=\text { No fue madre } \\
\text { adolescente }\end{array}$ & & $(1.020-1.439)$ & $(1.006-1.454)$ \\
\hline $\begin{array}{l}\text { Escolaridad Más de } \\
\text { secundaria (IC 95\%) }\end{array}$ & & $1.165^{\star}$ & 1.137 \\
\hline $\begin{array}{l}\text { Ref }=\text { Secundaria } \\
\text { o menos }\end{array}$ & & $(0.985-1.377)$ & $(0.943-1.371)$ \\
\hline $\begin{array}{l}\text { Número de hijos } \\
3+\text { (IC 95\%) }\end{array}$ & & 0.880 & \\
\hline Ref $=1-2$ hijos & & $(0.745-1.040)$ & \\
\hline$-2 \mathrm{LL}$ & 3400.440 & 3394.747 & 2754.730 \\
\hline
\end{tabular}

\section{Conclusión}

La información sobre la sexualidad y los métodos anticonceptivos podríamos decir que, en cierta medida están al alcance de los adolescentes mexicanos hoy en día. Sin embargo, la calidad de dicha información y el uso de anticonceptivos entre los y las adolescentes están aún, en más de un sentido, limitado por las restricciones impuestas, entre otras cosas, por su 
Apertura hacia la anticoncepción adolescente entre los padres y las madres en México: un estudio de caso ... / N. OJEDA et al.

ámbito familiar y en particular por sus propios padres y madres; por ser estos últimos quienes pueden facilitar o entorpecer la información, el acceso (y por consiguiente el uso) de métodos anticonceptivos entre la población adolescente. Los hallazgos obtenidos en este estudio al respecto, revelan una brecha entre los padres de distinto sexo en sus actitudes o posturas respecto de informar a los hijos e hijas adolescentes acerca de la sexualidad y la anticoncepción y de hacerlo con equidad de género. Las madres declaran posturas más favorables que los padres en estos temas, resumiéndose en una clara mayor apertura hacia la anticoncepción adolescente y la comunicación acerca de la sexualidad con los hijos y las hijas. En cambio, los padres varones presentan posturas más conservadoras en estas áreas que se resumen en una menor apertura hacia proporcionar información a los y las hijas adolescentes, pero en particular a estas últimas, sobre de la sexualidad y la anticoncepción.

Los resultados obtenidos también señalan que los padres de ambos sexos que tienen al menos estudios de secundaria muestran una mayor apertura hacia la sexualidad y la anticoncepción adolescente que aquellos con menor escolaridad. Otra característica que mostró ser estadísticamente significativa a favor de una mayor apertura en estos temas es el tener antecedente de maternidad adolescente, en la historia reproductiva de las madres, y de las esposas o compañeras conyugales de los padres varones. O sea, aquellos adultos que, de manera directa o bien indirecta, han vivido la experiencia de la maternidad adolescente consideran que debe haber más apertura hacia la sexualidad y la anticoncepción adolescentes.

La reflexión acerca de las posibles implicaciones del conjunto de estos hallazgos sobre la socialización en casa de los niños y adolescentes en materia de sexualidad y anticoncepción nos lleva a considerar un aspecto preocupante. Éste se refiere al consecuente ambiente confuso que, de manera involuntaria y tal vez inconsciente, se pudiera estar generando a consecuencia de las mencionadas diferencias entre papá y mamá en el proceso de socialización (vía la comunicación y actitudes) de los padres hacia sus hijos e hijas. Situación que también es extensiva a las familias con padres que no comparten el hogar con sus hijos pero que interactúan de manera regular. Al parecer son muchos los niños que pudieran estar recibiendo mensajes verbales y no verbales contradictorios respecto a la sexualidad y la anticoncepción por parte de sus mamas y papas. Esto es particularmente preocupante debido al impacto que tales contradicciones pudieran estar teniendo, en la exposición de los adolescentes al riesgo de contraer enfermedades de trasmisión sexual y la ocurrencia de embarazos a corta edad entre 
los adolescentes sexualmente activos ya sea de manera ocasional o bien regularmente y muchas veces sin el conocimiento de sus padres y madres. De tal modo, habría de considerarse la conveniencia de educar en materia de sexualidad y anticoncepción no solo a los adolescentes de ambos sexos, sino también a sus padres y madres, pero sobre todo a los primeros.

Por último, importa mencionar la conveniencia de realizar otros estudios sobre este mismo tema que permitan ampliar el conocimiento sobre el tema, particularmente en lo que a su dimensión cualitativa se refiere, ya que solo de este modo se podrá seguir avanzando en la implementación de políticas públicas de tipo social y de salud pública en materia de anticoncepción adolescente en el país.

\section{REFERENCIAS BIBLIOGRÁFICAS}

Aguilar, Jiménez María Eugenia, Ojeda, Norma e Islas Limón, Julieta Y., 2019, "Independencia del ingreso económico materno y su relación con la socialización de género con hijos e hijas, en Culturales, 7, e358. doi:https://doi.org/10.22234/ recu.201907001.e358

Aguilar, Jiménez María Eugenia, 2014, Estrategias para acceder al aborto en un contexto de penalización absoluta. Estudio de caso en Baja California, Tesis de Maestría en Estudios de Población, México: El Colegio de la Frontera Norte.

Brown, Susan y G. K., 2010, “Why don't teenagers use contraception? a qualitative interview study", en The European Journal of Contraception and Reproductive Health Care, June, (15), 197-204.

Casique, Irene, 2011, "Conocimiento y uso de anticonceptivos entre los jóvenes mexicanos: el papel del género", en Estudios Demográficos y Urbanos, 6 (3), 601 637.

Chandra-Mouli, V., Mcarraher, D. R., Philips, S. J., Williamson, N. E., and Hainsworth, G., 2014, "Contraception for adolescents in low and middle income countries: needs, barriers, and access", en Reproductive Health 11:1, doi:10.1186/1742-4755-11-1. Disponible en http://www.reproductive -health-journal.com/content/11/1/1.

Elkin, F. y Handel, G., 1984, "The processes and outcomes of socialization", en Elkin, F. y Handel, G., The Child and Society: The Process of Socialization. Nueva York: Random House, pp. 33-76.

Gayet, Cecilia, 2014, "El inicio sexual en México: retos en la prevención”, en Coyuntura Demográfica, (6), 43-49.

González, Humberto, 2011, “Caracterización sociodemográficas de la población en tránsito a la vida adulta en Tijuana”, Ojeda, N. y Zavala-Cosío, M. (eds.) Jó- 
Apertura hacia la anticoncepción adolescente entre los padres y las madres en México: un estudio de caso ... / N. OJEDA et al.

venes Fronterizos / Border Youth: Expectativas de Vida Familiar, Educación y Trabajo Hacia la Adultez, (pp. 57-78). México: El Colegio de la Frontera Norte.

Inkeles, A., 1968, “The needs of society and the process of socialization”, en Clausen, J.A. (coord.) Socialization and Society (pp. 75-129). Boston: Little, Brown and Company.

Islas, Limón Julieta Yadira, 2017, “Contradicciones en la incorporación de la perspectiva de género en la práctica docente". Trabajo presentado en el Seminario Roles de Género y Sexualidad: Socialización en Casa y Escuela. El Colegio de la Frontera Norte y Universidad Autónoma de Baja California, 2 de junio, 2017 (trabajo mimeográfico).

Juárez, Fátima y Gayet, Cecilia, 2005, "Salud sexual y reproductiva de los adolescentes en México: un nuevo marco de análisis para la evaluación y diseño de políticas", en Papeles de Población, 11 (45), 177-219.

Marini, M. M. y Fan, P.L., 2000, "Influences on gender-role attitudes during the transition to adulthood", en Social Science Research, 29, pp. 258 - 283.

Menkes, Catherine y Suárez, Leticia, 2013, "El embarazo de los adolescentes en México: ¿Es deseado?”, en Coyuntura Demográfica, (4), 21-28.

Menkes, Catherine, Suárez, Juárez, Leticia F. y Pérez, A. (eds), 2015, Salud reproductiva de los estudiantes de las escuelas de educación secundaria y media superior en el Distrito Federal. Mexico: CRIM-UNAM.

Ojeda, Norma, 2001, The Two Californias: A Binational Survey of Attitudes Regarding Family Planning and Abortion: A bi-linguial report. San Diego, California: Planned Parenthood of San Diego and Riverside Counties.

Ojeda, Norma, 2013, “Cambios y continuidades de la unión libre en México: el caso de los jóvenes en Tijuana”, en Perfiles Latinoamericanos, 21 (42), 193-224.

Ojeda, Norma y González Ramírez, Raúl, 2019, “Actitudes de padres mexicanos acerca de la igualdad de género en los roles y liderazgos familiares", en Estudios Demográficos y Urbanos vol. 34, núm 1 (100), enero-abril, pp. 169 - 219.

Palma, Cabrera Yolanda, 2019, La salud reproductiva de las adolescentes en Baja California: resultados de una encuesta de hogares. México: El Colegio de la Frontera Norte (en prensa).

Palma, Cabrera Yolanda y Reding, Arturo, 2011," Características del inicio de la vida sexual y reproductiva de las jóvenes en Tijuana", en Ojeda, N. y Zavala-Cosío, M. (eds.) Jóvenes Fronterizos / Border Youth: Expectativas de Vida Familiar, Educación y Trabajo Hacia la Adultez, (pp. 79-100). México: El Colegio de la Frontera Norte.

Ramos Vargas, Alina, 2016, Socializar en sexualidad: perspectivas de madres y padres de familia en Tijuana. Tesis de Maestría en Estudios de Población, México: El Colegio de la Frontera Norte. 
Rojas, Olga y Castrejón, Jose Luis, 2018, “Género e iniciación sexual en México. Detección de diversos patrones por grupos sociales", en Estudios Demográficos y Urbanos, vol. 26, núm. 1 (76) 75 - 111.

Soto, S., M. Osorio, Rodríguez, R. y Pérez, N., 2014, “Comportamiento sexual y anticoncepción en la adolescencia”, en Acta Pediátrica de México, 35 (6), 490498.

Stern, Claudio y García, Elizabeth, 2001, "Hacia un nuevo enfoque en el campo del embarazo adolescente", en Stern, C. y Figueroa, J. (eds.) Sexualidad y salud reproductiva. Avances y retos para la investigación (pp. 331-358). Mexico: El Colegio de México.

Welti, Carlos, 2005, "Inicio de la vida sexual y reproductiva", en Papeles de Población, 11 (45), 143-176.

Zavala, María Eugenia y Páez, O., 2013, "El retraso en la salida de la escuela no pospone la maternidad en México”, en Coyuntura Demográfica (4), 13-19.

\section{RESUMEN CURRICULAR DE LAS AUTORAS}

\section{Norma Ojeda de la Peña}

Es doctora en sociología por la Universidad de Texas en Austin, maestra en demografía por El Colegio de México y licenciada en sociología por la Universidad Nacional Autónoma de México. Es miembro del Sistema Nacional de Investigadores desde 1988. Ha recibido varios reconocimientos académicos entre los que se encuentran las distinciones Fulbright-García Robles y Fulbright Border Scholar. Ha realizado investigaciones financiadas por prestigiosas fundaciones mexicanas y extranjeras como son: CONACYT, Fundación John D. and Catherine McCarthur y Fundacion Ford. Ha publicado más de sesenta manuscritos en forma de libros, libros editados, artículos en revistas especializadas y capítulos de libros arbitrados en los temas de formación y disolución familiar, salud reproductiva de las mujeres y género en la socio demografía. Actualmente es profesora del Departamento de Sociología en San Diego State University e investigadora asociada del Departamento de Estudios de Población en el Colegio de la Frontera Norte.

Dirección electrónica: nojeda@colef.mx

Registro ORCID: http://orcid.org/0000-0002-8009-3096

\section{Raúl Sergio González Ramírez}

Es Doctor en Sociología por The University of Texas at Austin, Maestro en Demografía por El Colegio de México, y Licenciado en Estadística Social 
Apertura hacia la anticoncepción adolescente entre los padres y las madres en México: un estudio de caso ... / N. OJEDA et al.

por la Facultad de Economía de la Universidad Autónoma de Nuevo León. Actualmente es profesor investigador del Departamento de Estudios de Población de El Colegio de la Frontera Norte. Sus líneas de investigación incluyen los estudios de los componentes demográficos en la frontera entre México y Estados Unidos, salud y envejecimiento poblacional, así como los métodos estadísticos para el estudio de los fenómenos sociales y de la salud poblacional. Miembro del Departamento de Estudios de Población de El Colegio de la Frontera Norte. Recibió el Premio Nacional de Demografía en 2014. Dirección electrónica: raul@colef.mx

Registro ORCID: http://orcid.org/0000-0002-7380-5067

\section{Alina Alejandra Ramos Vargas}

Es Maestra en Estudios de Población por el Colegio de la Frontera Norte y Licenciada en Psicología por la Universidad Autónoma de Baja California. Sus principales áreas de trabajo son la investigación y docencia en ciencias de la salud y educativas. Ha impartido cursos de Metodología de la investigación y Psicología. Publicaciones recientes: coautora del capítulo "Evaluación del diseño de la prueba de matemáticas de primaria (06) del PLANEA ELCE 2015" en el libro Apuntes de Investigación Educativa, Qartuppi, abril, 2019, ISBN 978-607-98270-9-0. Actualmente es Docente en la Preparatoria Federal Lázaro Cárdenas, perteneciente a la Subsecretaria de Educación Media Superior, Dirección General de Bachillerato.

Dirección electrónica: alina.ramos@lazarocardenas.edu.mx Registro ORCID: http://orcid.org/0000-0002-4319-4912. 